\title{
Agroforestería para la conservación del suelo y otros recursos naturales
}

\author{
Agroforestry for soil conservation and other natural resources \\ Ruiz Velez Jessica Fernanda ${ }^{1}$ \\ ${ }^{1}$ Licenciada en Producción Agropecuaria, Universidad de los Llanos \\ agroforesteria@unillanos.edu.co
}

Recibido 05 de Marzo 2013, Aceptado 15 de Abril 2013

\section{RESUMEN}

Una definición de agroforestería que abarca varios aspectos es: ciencia interdisciplinaria, con tradición, innovación productiva y conservación de la naturaleza donde existen formas de manejo y aprovechamiento de sistemas y desarrollo de saberes tradicionales y novedosos, fortalecimiento de la identidad cultural, interacciones ecológicas totales de complementariedad del sistema, diversificación del paisaje, uso adecuado de recursos naturales, privilegio del trabajo humano, utilizando tecnologías de bajo impacto ecológico y en la que se presentan relaciones sociales, ambientales y económicas de bienestar, equidad y justicia. El hecho de disminuir las hectáreas de bosque en Suramérica, ha generado perdida de la biodiversidad, degradación de los recursos naturales (agua, aire y suelo), y desplazamiento de algunas tribus indígenas, lo que ha ocasionado una baja en la productividad agrícola y animal, y como si fuera poco, un alto índice de pobreza, de las poblaciones de campesinos que están dedicados a estas actividades. Lo anterior se debe, a la costumbre que poseen los países suramericanos, de poner en práctica modelos con una alta dependencia de agroquímicos, donde los sistemas de producción se basaron en monocultivos, con la importación de paquetes tecnológicos, implementación de maquinaria agrícola no apta para el trópico; que contribuyeron a empeorar la crisis ambiental por la que hoy estamos pasando. Se han planteado una serie de argumentos, orientados hacia la sostenibilidad considerando estrategias para la conservación de recursos naturales, y energía en su ciclicidad; en lo cual la "Agroforestería", desempeñaría un papel importante debido a la gran variedad de especies arbóreas que existen 
en el trópico de Suramérica, buscando con ello sistemas amigables con el medio ambiente y así brindar una calidad de vida óptima para los campesinos y su entorno, ya que aumenta la productividad animal y vegetal, así como el mejoramiento de los paisajes.

Palabras clave: Sostenibilidad, sistemas agroforestales.

\begin{abstract}
A definition of agroforestry that covers various aspects is interdisciplinary science with tradition, innovation production and conservation of nature where there are forms of management and use of systems and development of traditional and novel, strengthening cultural identity, total ecological interactions complementarity system, landscape diversification, proper use of natural resources, human labor privilege, using technologies with low environmental impact and which are social, environmental and economic welfare, equity and justice. The fact decrease hectares of forest in South America, has produced loss of biodiversity, degradation of natural resources (water, air and soil), and displacement of indigenous tribes, which has caused a decline in agricultural and animal productivity, and last but not least, a high poverty rate, rural populations who are dedicated to these activities. This is due, custom to have the South American countries, to implement models with a high dependence on agrochemicals, where production systems are based on monocultures, with imported technology packages, implementation of agricultural machinery unsafe the tropics, which contributed to the worsening environmental crisis which we are now passing. A series of arguments have been raised, oriented towards sustainability considering strategies for the conservation of natural resources, and energy in its cyclicality, in which the "agroforestry", play an important role due to the large variety of tree species found in the tropics of South America, seeking to friendly systems environment and so provide an optimal quality of life for farmers and their environment, increasing plant and animal productivity and improving landscapes.
\end{abstract}

Keywords: Sustainability, agroforestry system. 


\section{INTRODUCCIÓN}

Suramérica, es rica en su biodiversidad que se encuentra distribuida en diferentes tipos de terrenos que en su mayoría son aptos para la producción agropecuaria; parte de ellos, se encuentran ubicados en el trópico ecuatorial, y es allí donde se sitúan algunas zonas boscosas; pero debido al constante proceso en el cambio de uso de suelos para la ganadería, donde se han establecido grandes zonas para el cultivo de pastizales, adicionalmente se están implantando la producción intensiva de biocombustibles, los bosques con que cuenta, están siendo afectados, debido a la tala descontrolada, aumentándose la cantidad de sabanas, que prácticamente son en $80 \%$ monocultivos, lo cual no favorece desde ninguna perspectiva la conservación de la flora y la fauna en detrimento de las poblaciones nativas, que habitan determinada región (OLADE, 2003).

El hecho de disminuir las hectáreas de bosque, ha generado perdida de la biodiversidad, degradación de los recursos naturales (agua, aire y suelo), y desplazamiento de algunas tribus indígenas, lo que ha ocasionado una baja en la productividad agrícola y animal, y como si fuera poco, un alto índice de pobreza, de las poblaciones de campesinos que están dedicados a estas actividades. Lo anterior se debe, a la costumbre que poseen los países suramericanos, de poner en práctica modelos con una alta dependencia de agroquímicos, donde los sistemas de producción se basaron en monocultivos, con la importación de paquetes tecnológicos, implementación de maquinaria agrícola no apta para el trópico; que contribuyeron a empeorar la crisis ambiental por la que hoy estamos pasando (FAO, 2009).

Sin embargo, es de considerar, que en la actualidad, por las consecuencias que hemos generado con nuestras acciones, el hombre ha comenzado un proceso de toma de conciencia, y ha ideado una serie de estrategias, llamadas según Delgado, (2004) medidas agronómicas para la conservación del suelo, donde se considera manejo de la vegetación, rotación y asociación de cultivos, coberturas de tipo mulch, labranza de conservación y agroforestería, siendo esta última, la que mejor se adapta a las condiciones del trópico. 
En su proyecto de desarrollo económico CEPAL/GTZ, (2003) han planteado una serie de argumentos, orientados hacia la sostenibilidad planteando estrategias para la conservación de los recursos naturales, y la energía en su ciclicidad; en lo cual la "Agroforestería", desempeñaría un papel importante debido a la gran variedad de especies arbóreas que existen en el trópico de Suramérica, buscando con ello sistemas amigables con el medio ambiente y así brindar una calidad de vida óptima para los campesinos y su entorno, ya que aumenta la productividad animal y vegetal, así como el mejoramiento de los paisajes.

\section{ANÁLISIS DE LA PROBLEMÁTICA}

El mundo se encuentra en una alta dependencia de combustibles fósiles, un mal manejo de la tierra, y un acelerado incremento de la población, lo cual ha ocasionado una disminución en la diversidad biológica y además ha contribuido en la degradación de los recursos naturales, que hacen posible el sostenimiento de la vida. Una de las causas principales de degradación del ambiente, es la tala de bosques con el fin de ser convertidos en áreas de producción agropecuaria, por ejemplo, en Colombia se talan anualmente de 360 a 600 mil hectáreas de bosque, debido principalmente a procesos de colonización, la utilización de la leña como recurso energético y la expansión de la industria forestal. Estos procesos llevan consigo la pérdida de la diversidad biológica, la alteración de los sistemas hídricos, la erosión de los suelos y la pérdida del equilibrio ecológico global (FAO, 2009).

En Colombia, tratando de maximizarse la rentabilidad de las empresas pecuarias, se ha optado por producir intensivamente, y según cifras del Paredes y Mojica, (2005) la producción avícola ha ascendido del año 1990 con un numero de pollos de engorde de 223 millones a 485 millones en el 2004. El DANE, (2002) reporta que la ganadería ascendió del año 2002 de 3.275 .799 cabezas de ganado a 3.926.628 en el 2006; este hecho genera "un dramático aumento en las emisiones atmosféricas de amoniaco, lo cual se ha reportado en los últimos años en las zonas de agricultura intensiva de animales. Lo descrito se ve representado, en las Gráficas 1, 2 y 3, adaptadas de Coma y Bonet, (2004); Gay y Knowlton, (2005). 

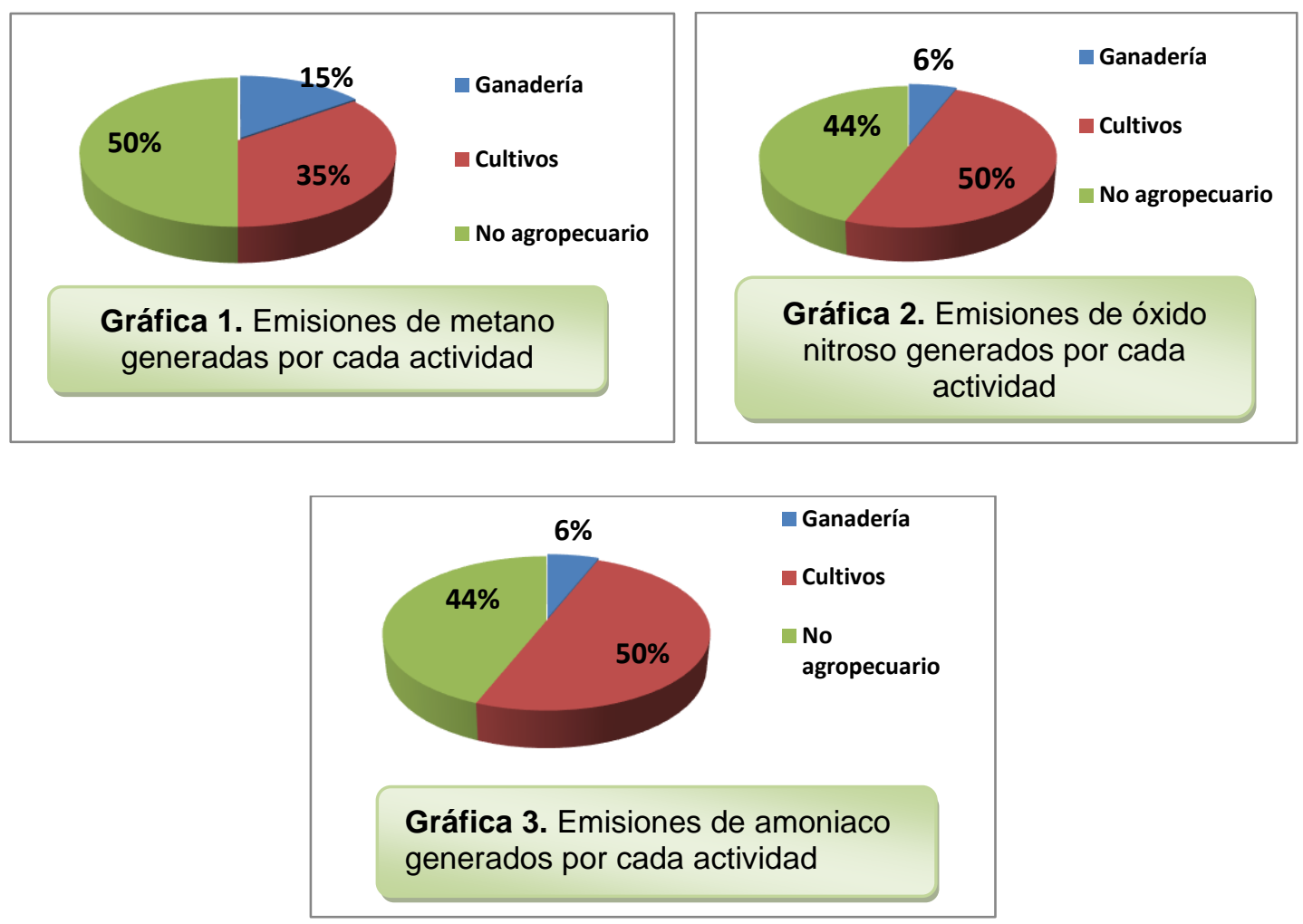

Otra de las causas, de degradación del ambiente, es la producción agrícola, que utiliza el $72 \%$ del agua para consumo, y la cual es contaminada por los agroquímicos aplicados durante los ciclos productivos de los cultivos. De acuerdo a lo anterior, el problema consiste en las formas de producción implementadas a partir de la revolución verde y los modos de vida tan arraigados que no generan sostenibilidad, ni conservación, sino por el contrario producen un ambiente mal sano, (Coma y Bonet, 2004).

\section{¿QUE ES LA AGROFORESTERÍA?}

Para poder hacer referencia a ella como una alternativa mucho más eficiente de producción, conservación del suelo y otros recursos naturales, es menester conocer algunos enfoques, como el que hace referencia a sistemas y tecnologías de uso del suelo en los cuales las especies leñosas perennes (árboles, arbustos, palmas, y otros) se utilizan deliberadamente en el mismo sistema de manejo con cultivos agrícolas y producción animal, en alguna forma de arreglo espacial o secuencia temporal (Nair, 1985; Ospina, 2003). 
Petit, (1993) y Ospina, (2000) afirman que es un sistema de producción o formas de uso y manejo de los recursos naturales en los que las especies leñosas (árboles, arbustos y palmas), son utilizadas en asociación deliberada con cultivos agrícolas o animales en el mismo terreno, de manera simultánea o en secuencia temporal.

Palomeque, (2009) lo define como una ciencia interdisciplinaria, también una tradición e innovación productiva y de conservación de la naturaleza, desarrollada fundamentalmente por culturas agroforestales en tierras tropicales, donde existen formas de manejo y aprovechamiento de sistemas y desarrollo de saberes tradicionales y novedosos, fortalecimiento de la identidad cultural, interacciones ecológicas totales de complementariedad del sistema, diversificación del paisaje, uso adecuado de recursos naturales, privilegio del trabajo humano, utilizando tecnologías de bajo impacto ecológico y en la que se presentan relaciones sociales, ambientales y económicas de bienestar, equidad y justicia.

\section{CLASIFICACIÓN DE LOS SISTEMAS AGROFORESTALES}

Los sistemas agroforestales se clasifican según Correa et al., (2006) en: silvopastoriles (árboles asociados con ganadería), agrosilvoculturales (árboles combinados con cultivos), agropastoriles (cultivos combinados con ganadería) y sistemas agrosilvopastoriles (árboles con cultivos y ganadería). En la zona tropical de Suramérica existen gran variedad de gramíneas, leguminosas y otras especies (Tabla 1) que tienen alta aceptación por parte del ganado por su alto contenido nutricional en proteína, calcio y fósforo, además han demostrado gran rusticidad y elevada tasa de producción de biomasa, lo cual hace que, en muchas producciones pecuarias, las utilicen como bancos forrajeros para complementar la alimentación de los animales en pastoreo. Se ha demostrado que algunas especies no leguminosas están en la capacidad de mejorar las condiciones del suelo, aunque no es del todo claro, como hacen sí, asociándose con hongos formadores de micorrizas que capturan fósforo o ácidos orgánicos en las raíces para permitir la asimilación de nitrógeno, tal cual lo hacen las leguminosas (Jama et al., 2000). 
Tabla1. Algunas especies que se pueden asociar en los sistemas agroforestales

\begin{tabular}{ll}
\hline Nombre común & Nombre científico \\
\hline Gramíneas & Panicum máximum \\
Pasto guinea o india & $\begin{array}{l}\text { Braciaria brizantha } \\
\text { Pasto marandú }\end{array}$ \\
Pasto amargo o común & Brachiaria decumbens \\
Pasto la libertad & Sacharum officinarum \\
Caña forrajera & \\
Leguminosas & \\
Maní forrajero & Arachis pintoi \\
Capica & Stylosanthes capitata \\
Matarratón & Gliricidia sepium \\
Cachimbo & Erithryna fusca \\
Guandul & Cajanus cajan \\
Acacia Roja & Delonix regia \\
Leucaena & Leucaena leucocefhala \\
Cratilya & Cratilia argéntea \\
Otras especies arbóreas & \\
Nacedero & \\
Achupalla & Trichanthera gigantea \\
Botón de oro & Tournefortia sp \\
Acalifa & Thitonia Diversifolia \\
Cayeno & Acalypha macrostacya \\
Guácimo & Hibiscus rosa-sinensis \\
\hline Fuente: & Guazuma ulmifolia \\
\hline
\end{tabular}

Fuente: Otálvaro, 2001; Lemus y Lemus 2004

\section{JUSTIFICACIÓN DE LA AGROFORESTERÍA EN LOS SISTEMAS AGROPECUARIOS}

América Latina goza de una vasta zona tropical, donde se posee una gran riqueza de biodiversidad, y además es territorio clave para la expansión de la frontera agrícola y ganadera; pero para que esto suceda, existen unos factores determinantes en la producción tropical, como lo es el clima, el suelo y los recursos naturales. El clima, modelado por la cordillera de los andes, determina un 
régimen de lluvias bastante amplio, y con ello un vasto número de fuentes de agua dulce, aptas para la agricultura, la alimentación humana y animal, por otro lado, la zona de trópico se caracteriza por tener una marcada intensidad lumínica, lo que permite una variada gama de especies vegetales, a lo que se suma, el recurso suelo, el cual es acido y casi infértil; según CEPAL/GTZ, (2003) en América tropical el $33.6 \%$ de los suelos potencialmente agrícolas son Oxisoles y el $21.4 \%$ son Ultisoles; y estos suelos son los que sostienen los sistemas de pastoreo extensivo, que extraen los pocos nutrientes con que cuenta el sustrato; ahora bien es de analizar, que este sistema bastante frágil, y con el aumento de la población, y el agotamiento de combustible fósil, es necesario implementar sistemas productivos mucho más eficientes, para suplir las necesidades actuales (Clapperton, 2003).

Con lo anterior se plantea que no se debe seguir implementado el sistema de producción que propone la revolución verde, pues "Este paradigma dio paso, en los países tropicales a la implantación de sistemas de producción con base en monocultivos, a la dependencia de un número reducido de especies vegetales y animales, a la importación de paquetes tecnológicos y a un uso elevado de insumos, producidos en países industrializados; lo que ha ocasionado un alto impacto negativo en el medio ambiente, debido al uso indiscriminado de los agroquímicos, los cuales aceleraron los procesos de deterioro y agotamiento de los recursos naturales, por lo tanto, está más que justificado implementar nuevos sistemas de producción, como la "Agroforesteria", la cual propone, el aumento de la productividad, diversificando ingresos, conservando el medio ambiente y satisfaciendo las necesidades de las personas (Nair, 1993; Ospina 2000).

De acuerdo con Ospina, (2003); Lemus y Lemus, (2004) y Funes, (2007) identifican las siguientes razones para que la "Agroforesteria" se integre a los sistemas agropecuarios en el trópico:

1- Permite diversificar ingresos: teniendo en cuenta que el mercado fluctúa, la diversificación puede significar una serie de entradas para la finca, pues no 
solo se tendría un cultivo, sino varios, que permitirían amortiguar los cambios de precio del mercado.

2- Aumenta la productividad: lo cual se demuestra en donde se especifica que en el sureste asiático se practica con éxito el pastoreo de bovinos y ovinos en plantaciones de palma de aceite y cocotero. La cantidad de luz transmitida bajo las plantaciones permite el desarrollo de pastos resistentes a un cierto nivel de sombra, entre ellos Panicum maximum y Brachiaria decumbens. La presencia de animales incrementa el reciclaje de nutrimentos y reduce la competencia del estrato herbáceo, mejorando la producción de coco. Los subproductos industriales de la extracción de aceite posibilitan el engorde de cerdos y pueden ser utilizados en la ceba de bovinos u ovinos.

3- Conserva energía y recursos hídricos: por ejemplo, existen árboles como el yopo (Anadenanthera peregrina), que sirve como fuente alterna de combustible para los asaderos. Además, cuando existe en un sistema diversidad de especies por estratos, se controla la escorrentía, la pérdida de suelo y además se adhieren a las raíces contaminantes que arrastran las aguas de lluvia, evitando que caigan a las fuentes de agua.

4- Crea un medio ambiente saludable: porque no solo se produce, sino que se mejora el suelo, el aire, el agua, y se crea un ambiente propicio para variedad de animales y plantas.

5- Satisface las necesidades: al mantener mayor biodiversidad en el entorno ambiental y productivo, contribuye en la mejora de la calidad de vida de las personas, representado esto en bienestar.

6- Recupera la capacidad productiva del suelo: existe un aporte de nitrógeno al mismo tiempo, a través de leguminosas arbóreas y arbustivas, además disminuye la pérdida de suelo por la erosión hídrica y eólica, debido a que la especies arbóreas presentan un sistema radicular mucho más profundo, que las gramíneas, lo cual les permite captar agua y minerales en perfiles del suelo más profundos, manteniendo el perfil superficial del suelo en buenas condiciones, que puede ser utilizado para otros cultivos. 
7- Reduce la velocidad del viento y mantiene temperatura del suelo: por efecto de las barreras vivas, disminuye hasta en $20 \%$ la tasa de evaporatranspiración y la temperatura en el suelo, aumentando la cobertura vegetal mediante la incorporación de hojarasca y abundancia de raíces, y de esta forma se mitigan los efectos del estrés de sequía en los cultivos, permitiendo a la vez un mayor aporte de nutrientes, y mejoramiento en la estructura y porosidad del suelo.

8- Aumento de diversidad: dando las condiciones adecuadas para el bienestar de la flora y fauna nativa, lo cual contribuye a la disminución de plagas, pues se fomentan los depredadores, que generalmente se constituyen en aves e insectos benéficos; además el recurso arbóreo, aporta sombra a los animales y a otros cultivos como café y cacao, aumentando la productividad de estos componentes.

\section{SITUACIÓN DE LA AGROFORESTERÍA EN LA ORINOQUIA COLOMBIANA}

El desarrollo de la agroforestería en Colombia, comienza en la década de los ochenta, pero en los años 90, fue donde se generó una toma de conciencia sobre la importancia del árbol en el desarrollo rural. Bajo esta perspectiva, varias instituciones de carácter gubernamental y no gubernamental comenzaron a fomentar la agroforestería como alternativa sostenible y competitiva para el desarrollo agropecuario de diversas zonas del país. Lo expuesto, va en pro de las nuevas políticas que propone el gobierno nacional, quienes han presentado el más importante proyecto de recuperación de bosque tropical húmedo del mundo en la región de la Orinoquia (Cano y Arias, 2004).

"Este megaproyecto apunta a crear el sumidero de dióxido de carbono de mayor magnitud del mundo en su historia reciente, con un potencial de fijación del mismo de 25 toneladas por hectárea por año a través de la reforestación y la consecuente recuperación de la capa vegetal, dando iniciativas complementarias en el ámbito agrícola de producción limpia y ambientalmente compatible de alimentos. Es decir, un potencial de secuestro de dióxido de carbono de 7.908 millones de toneladas durante un período de 50 años (Cano y Arias, 2004). 
De esta manera Colombia está empeñada en aproximarse al cumplimiento de los objetivos del Protocolo de Kyoto, y de las metas del milenio tendientes a la reducción de la pobreza en el mundo a menos del 50\% para el año 2015, proclamada en la Cumbre de la tierra, para el desarrollo sostenible (ONU, 2002).

En consideración con lo anterior, Colombia, está apuntando a desarrollar una agricultura tropical, orientada a conocer y aprovechar las relaciones que se establecen en los sistemas de producción agropecuarios y el componente arbóreo, con el propósito de incrementar nuevos productos promisorios como los biocombustibles y los tendientes a proporcionar seguridad alimentaria de manera orgánica e inocua.

Para la planificación territorial en Colombia, se ha identificado como prioridad, la realización de acciones, que a través de los sistemas agroforestales y las plantaciones protectoras-productoras, propendan por la recuperación y manejo de micro-cuencas en las márgenes y cabeceras de los ríos, para aumentar la cobertura vegetal de áreas degradadas y de esta manera devolver la capacidad productiva de suelos erosionados. Se ha demostrado que, en las plantaciones forestales de zonas bajas, el pastoreo de las arvenses que están dentro de éstas, contribuye con el pago de la mitad de los costos para el mantenimiento de los árboles, además controla la invasión de pastos dejando utilidades adicionales, (Correa et al., 2006).

Experiencias con unidades familiares campesinas de minifundio, las cuales combinan diferentes sistemas pecuarios: ganado, cerdos y aves con policultivos forrajeros de corte y acarreo como: Trichanthera gigantea, Erytrina fusca, Tithonia diversifolia, Erytrina rubrinervia, Urera sp, Boehmeria nivea, Leucaena leucocephala, Alocassia macroriza y caña de azúcar) generan un balance anual $46.9 \%$ superior (1977 Vs US \$1050/año) comparadas con unidades familiares que utilizan tecnologías convencionales de pastoreo extensivo y concentrados (Murgueitio, 1999). 


\section{CONCLUSIONES}

La agroforestería es una táctica productiva, muy apta para el trópico, pues se encamina a manejar los recursos naturales de forma eficiente, buscando un equilibrio y evitando el uso indiscriminado de agroquímicos y maquinaria agrícola pesada; asimismo porque es en el trópico donde se presenta deforestación de los bosques y selvas (Amazonas) por la expansión agrícola y pecuaria actual. Además, es de considerar que los índices de pobreza son altos, por la mala implementación de los cultivos (monocultivos), y por la falta de estrategias y soluciones que conlleven a obtener un desarrollo sostenible.

Es importante mencionar, que cuando hay mayor cantidad de componentes en un sistema, las pérdidas de energía se reducen, lo cual se logra cuando en una finca se implementan los sistemas agroforestales, y que además de dicha ayuda en gran medida ayuda a la conservación del suelo, no excluye al ser humano del sistema, sino que lo integra y le proporciona una mejor calidad de vida, en cuanto a salud, bienestar, recreación, y seguridad.

"Es de humanos errar, pero también es de los humanos la razón, que de poco o nada ha servido, cuando a nosotros mismos nos hemos acabado por la ambición y la codicia"

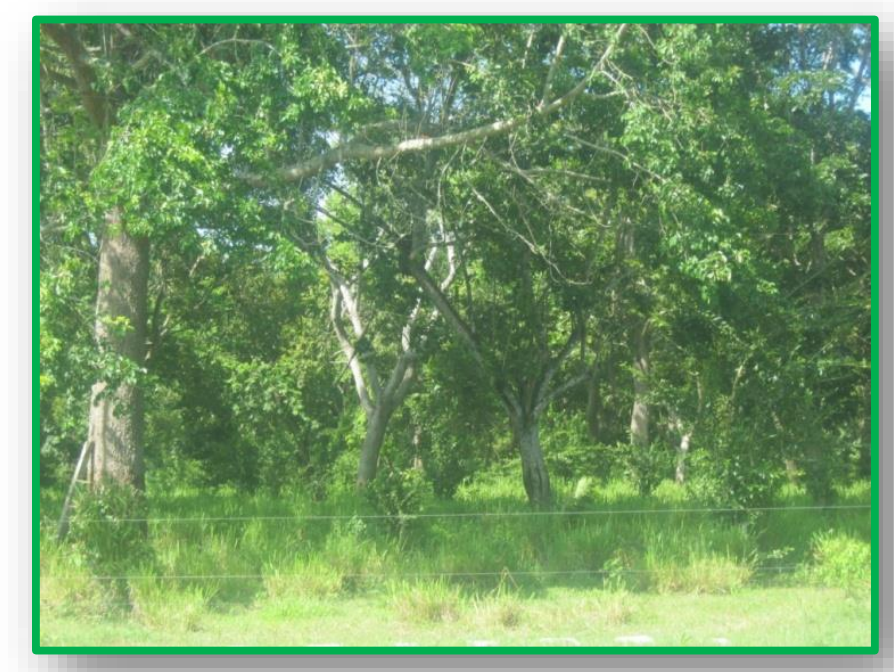

Fotografía 1. Los sistemas agroforestales propenden por la recuperación de suelo, agua y mejoramiento de las condiciones climáticas de un entorno. 


\section{BIBLIOGRAFÍA}

1. Cano C., Arias A. El renacimiento de la Orinoquia alta de Colombia: un megaproyecto para el mundo. Ministerio de Agricultura y Desarrollo Rural República de Colombia. 2004. Disponible En: http://www.minagricultura.gov.co/archivos/cartilla\%200rinoc0\%20completa.pdf

2. CEPAL/GTZ. Sostenibilidad energética en América Latina y el Caribe: el aporte de las fuentes renovables: Proyecto "Promoción del Desarrollo Económico en América Latina y el Caribe, por medio de la Integración de Propuestas de Políticas Ambientales y Sociales". 2003. Recuperado 3 de Noviembre $2012 . \quad$ Disponible En: http://www.eclac.org/publicaciones/xml/9/13319/Lcl.1966e.pdf

3. Clapperton, M. J. Increasing soil biodiversity through Conservation Agriculture - Managing the soil as a habitat. Proceedings II Congresso Mundial sobre Agricultura Conservacionista. p. 136-145. 2003.

4. Coma J., Bonet J. Producción ganadera y contaminación ambiental, XX Curso de Especialización FEDNA Barcelona, 22 y 23 de noviembre, p. 237-272. 2004.

5. Correa H. D., Ruiz, S. L., Arévalo, L. M. (eds). Plan de acción en biodiversidad de la cuenca del Orinoco-Colombia / 2005 - 2015 - Propuesta Técnica. Bogotá D.C.: Corporinoquia, Cormacarena, IAvH, Unitrópico, Fundación Omacha, Fundación Horizonte Verde, Universidad Javeriana, Unillanos, WWF - Colombia, GTZ - Colombia, Bogotá, D. C., 330 p. 2006.

6. DANE. Sacrificio consumo interno, información base censo 1983 - 1996. Año 1997, Muestra de Sacrificio de Ganado Vacuno en 67 municipios. Frigoríficos de exportación, conocimiento de embarque 1982-1990. ICA, Colombia. 2002.

7. Delgado F. Agricultura Sostenible y Mejoramiento de Suelos de Ladera. Serie Suelos y Clima SC- 76. CIDIAT. Mérida. Venezuela. 2004.

8. Edwards, C. A. Earthworms. Chapter 8 En: Soil Biology Primer. Soil and Water Conservation Society. Rev. Edition. Ankeny lowa. 2000.

9. FAO. Situación de los bosques en el mundo, División de Comunicación, Roma, Italia. 80 p. 2009.

10. Gay S., Knowlton K. Ammonia emissions and animal agriculture, Dairy Science, Virginia Tech, Publication Number 442-110, Posted March, 2005.

11. Funes $F$. Avances científicos en sistemas de producción agrosilvopastoriles en cuba. Centro internacional de agricultura tropical. (CIAT), Colombia, 2007.

12. Gobierno del Estado de Chiapas-Secretaría de Desarrollo Rural. Agroforestería. Guía de extensionista forestal II. 15 p. 2006.

13. Jama B., Palm C. A., Buresh, R. J., Niang A., Gachengo, C., Nziguheva G., Amadalo B. Tithonia diversifolia (Hemsl.) Gray as a green manure for soil fertility improvement in western Kenya: Review. Agroforestry Systems. 4: 201. 2000.

14. Lemus L., Lemus V. Plantas de uso forrajero en el trópico, cálido y templado de Colombia. Universidad de los Llanos programa de Ingeniería Agronómica, Villavicencio, Colombia. 360 p. 2004. 
15. Malagón R., Prager M. El enfoque de sistemas: una opción para el análisis de las unidades de producción agrícola. Universidad Nacional de Colombia, año 2001.

16. Marcos Z. Z. El suelo en los ciclos de la naturaleza, XIII Congreso Latinoamericano De La Ciencia Del Suelo, Brasil, 4-8 de agosto de 1996.

17. Mojica A., Paredes J. Características del sector avícola colombiano y su reciente evolución en el departamento de Santander, Centro Regional de Estudios Económicos del Banco de la República. 26 p. 2005.

18. Murgueitio E. Sistemas Agroforestales para la Producción Ganadera en Colombia. CIPAV Cali. Colombia. 1999. Disponible En: http://www.cipav.org.co/redagrofor/memorias99/Murgueit.htm

19. Nair P. Classification of agroforestry systems. Agroforestry Systems. 3 (2): 301-318. 1985.

20. Nair, P. K. An introduction to agroforestry. Kluwer Academic Publishers ICRAF. Dordrecht, The Netherlands. 1993.

21. OLADE (Organización Latinoamericana de Energía) Sistema de Información Económica-Energética/SIEE. Balances Energéticos de los países de América Latina y el Caribe. 2003. Recuperado 1 de noviembre 2012. Disponible En: http://www.olade.ec.org/siee

22. ONU. La Cumbre de la Tierra celebrada, Johannesburgo (África del Sur). Cumbre Mundial del desarrollo sostenible: del 26 de agosto al 4 de septiembre de 2002.

23. Ospina A. Contribución al conocimiento de los criterios de clasificación y caracterización de los sistemas agroforestales. Palmira, Monografía (Ingeniero Agrónomo). Universidad Nacional de Colombia, Sede Palmira. Facultad de Ciencias Agropecuarias y Fundación Ecovivero, 262 p. 2000.

24. Ospina A. Agroforestería: aportes conceptuales, metodológicos y prácticos para el estudio agroforestal. Cali, Colombia: ACASOC, 205 p. 2003.

25. Otalvaro N. Identificación y caracterización de árboles y arbustos con potencial forrajero en los Municipios de San Luis de Cubarral, El Dorado y Guamal (Meta). Tesis para optar al título de Médico Veterinario Zootecnista. Universidad de los Llanos, Villavicencio, Meta. 107 p. 2001.

26. Palomeque E. sistemas agroforestales. Huehuetán, Chiapas, México, Junio de 2009. Recuperado 30 de Octubre 2012. Disponible En: http://www.monografias.com/trabajospdf2/sistemas-agroforestales/sistemasagroforestales.pdf

27. Petit A. Una revisión sobre el concepto de agroforestería. En: Revista Forestal Latinoamericana. 12 (edición especial): 7-21. 1993. 